\title{
Ceftazidime-Avibactam as Salvage Treatment for Infections Due to Carbapenem-Resistant Klebsiella pneumoniae in Liver Transplantation Recipients
}

\author{
Fang Chen ${ }^{1,2, *}$ \\ Han Zhong ${ }^{1} *$ \\ Tengjiao Yang ${ }^{3}$ \\ Chuan Shen ${ }^{2}$ \\ Yuxiao Deng ${ }^{4}$ \\ Longzhi $\mathrm{Han}^{2}$ \\ Xiaosong Chen ${ }^{2}$ \\ Haomin Zhang ${ }^{5}$ \\ Yongbing Qian $\mathbb{D}^{2}$
}

'Department of Pharmacy, Renji Hospital, School of Medicine, Shanghai Jiao Tong University, Shanghai, People's Republic of China; ${ }^{2}$ Department of Liver Surgery, Renji Hospital, School of Medicine, Shanghai Jiao Tong University, Shanghai, People's Republic of China; ${ }^{3}$ Department of Pharmacy, The Third People's Hospital of Henan Province, Zhengzhou, People's Republic of China; ${ }^{4}$ Department of Critical Care Medicine, Renji Hospital, School of Medicine, Shanghai Jiao Tong University, Shanghai, People's Republic of China; ${ }^{5}$ Department of Laboratory Medicine, Renji Hospital, School of Medicine, Shanghai Jiao Tong University, Shanghai, People's Republic of China

*These authors contributed equally to this work

Correspondence: Haomin Zhang Department of Laboratory Medicine, Renji Hospital, School of Medicine,

Shanghai Jiao Tong University, Shanghai,

People's Republic of China,

Tel +86-2I-683837I5

Email zhanghaomin@renji.com

Yongbing Qian

Department of Liver Surgery, Renji

Hospital, School of Medicine, Shanghai

Jiao Tong University, Shanghai, People's

Republic of China,

Tel +86-2l-683837I5

Email qianyb79@hotmail.com
Background: Ceftazidime-avibactam (CZA) has been approved in vitro activity against carbapenem-resistant $K$. pneumoniae (CRKP), but the experience for the treatment of CRKP in liver transplantation (LT) recipients was limited, and previous data on its efficacy in this setting are lacking.

Methods: LT recipients with CRKP infection who received CZA treatment were reviewed retrospectively, microbiological and clinical response, adverse events were also assessed. The primary outcome was 30-day mortality after CZA administration.

Results: CZA was used in 21 LT recipients (including 4 pediatric patients) with CRKP infections after failure with other antimicrobials. CZA was administered as monotherapy in 4 patients. Median time from the onset of CRKP infection until the initiation of CZA treatment was 2 days (IQR, 1-6.5), and the median treatment duration was 12 days (IQR, 8.5-18.5). The mortality at 14 days, 30 days and all-cause was $28.6 \%, 38.1 \%$ and $42.9 \%$, respectively. In adult patients, clinical response of 14 days and 30 days was $70.6 \%(12 / 17)$ and $58.8 \%(10 /$ 17), respectively, while in pediatric patients the 14-day and 30-day clinical response were both $75 \%$, respectively. The relapse rate during the treatment developed in 3 patients after 30 days with the cessation of CZA monotherapy. CZA resistance was not detected in any case and $3(3 / 21,14.3 \%)$ patients developed acute kidney injury related to uncontrolled infection. Conclusion: CZA shows promising results, even in monotherapy, for the treatment of patients with severe infections due to carbapenem-resistant $K$. pneumoniae among LT recipients. The emergence of resistance to CZA was not observed.

Keywords: carbapenem-resistant Klebsiella pneumoniae, ceftazidime-avibactam, liver transplantation, intra-abdominal infection, pneumonia

\section{Introduction}

Carbapenem-resistant Klebsiella pneumoniae (CRKP) infection is becoming a very serious complication, especially after liver transplantation (LT). ${ }^{1-5}$ Due to limited options, CRKP infection is often associated with high morbidity and unfavorable outcomes, with overall mortality ranging from $40 \%$ to $70 \%{ }^{6-9}$ The rate of CRKP infection in liver transplantation recipients is higher than general population, ranging from $6 \%$ to $13 \%$, but in epidemic situations up to $23 \%$. $^{10-12}$ Some studies have reported that carbapenem resistance was an independent risk factor for mortality, possibly due to inappropriate initial antimicrobial treatment. ${ }^{13-15}$

Effective therapeutics for carbapenem-resistant Enterobacterales (CRE) infection is scarce. Recently, the antibiotics of polymyxins, tigecycline, fosfomycin and 
aminoglycosides have been used against CRE infection, however, concerns remain about increased drug resistance, limited efficacy and toxicity. ${ }^{16-18}$ Ceftazidimeavibactam (CZA) is a new $\beta$-lactam/ $\beta$-lactamase inhibitor, ${ }^{19}$ which has demonstrated in vitro activity against Klebsiella pneumoniae carbapenemases (KPCs) and OXA-48 producing Enterobacterales. ${ }^{20}$ Real-world experience of CZA in the treatment of infections caused by resistant gram-negative bacilli, including CRE infections is accumulating. ${ }^{21-24}$ Patients infected with CRE achieved good response to CZA treatment, with an overall success rate of about $70 \% .{ }^{25-27}$ Castanheira et $\mathrm{al}^{28}$ showed that the sensitivity of Enterobacterales to CZA was $99.9 \%$, and only 3 of $120 \mathrm{KPC}$-type carbapenemase producing strains developed resistance to CZA. Tumbarello et al reported a retrospective study from 22 hospitals in Italy for infections caused by KPC-Kp, CZA appeared to be an independent risk factor for 30-day allcause mortality for the treatment of serious KPC-Kp infections, even when used alone. ${ }^{29}$ However, most of the available data on the efficacy and safety of CZA in clinical applications were from observational studies. In addition, scarce data exists related to the efficacy of CZA for CRKP infections in patients after liver transplantation.

Thus, we conducted a retrospective study to analyze the effectiveness and safety of CZA treatment, which included 21 cases with CRKP infection after LT.

\section{Materials and Methods Study Design and Population}

A retrospective study was conducted on inpatients with carbapenem-resistant Klebsiella pneumoniae infection after LT, who had received CZA treatment for at least 72 hours between April 2018 and August 2021. During this period, CZA was administered for infections caused by confirmed CRKP organisms. A standard dosage of CZA ( $2 \mathrm{~g}$ of ceftazidime with $0.5 \mathrm{~g}$ of avibactam intravenously every $8 \mathrm{~h}$ over 2 hours) was administered to all included patients, with adjustments in patients with moderate or severe renal dysfunction.

\section{Ethics Statement}

The study was conducted in accordance with the Declaration of Helsinki (as revised in 2013). The protocol was reviewed and approved by the institutional review committee of Renji Hospital (Approval No: 2021-061-B) and individual consent for this retrospective analysis was waived. All livers were donated voluntarily with written informed consent. No donor organs were obtained from executed prisoners. It was conducted in accordance with the Declaration of Istanbul.

\section{Outcome and Explicative Variables}

Primary outcome was 30-day mortality after CZA administration. Secondary outcomes were 14-day mortality, microbiological outcome and adverse events related CZA.

The recorded variables included demographic characteristics (age, sex, and weight), underlying diseases, MELD or PELD score, CZA treatment (Dosage, duration and adverse effects), microbiological resistance, prior or concomitant antimicrobial therapies, infection characteristics (SOFA score, onset of infection, and source of infection) and empirical or definitive antibiotic treatment.

\section{Definitions}

The clinical outcomes were characterized as following: Response, complete disappearance of the patient's clinical signs and symptoms associated with infection, and/or elimination of the infection, with no recurrence within 30 days after onset of CZA treatment; Failure, continuation of signs and symptoms from baseline to the end of antibiotic treatment, and/or death due to infection and/or recurrent infection. Microbiological failure was defined as the isolation of CRKP from samples obtained from the same source of infection and/or blood cultures following $\geq 7$ days of CZA treatment. Combination therapy was defined as combining use of two or more antibiotics for at least 48 hours, including inhaled products (only in cases of respiratory infection), regardless of in vitro activity.

CRE is defined according to centers for Disease Control criteria by phenotypic resistance to carbapenem or the presence of carbapenem hydrolases. Types of CRE infections are classified according to the National Health care safety net criteria. ${ }^{27}$

Adverse events related to CZA were defined as any adverse effect that occurred from the initiation of CZA treatment to within 30 days after discontinuation of CZA treatment.

\section{Isolate Collection and Microbiological Investigation}

The final isolates of CRKP cultured before CZA administration from 21 patients were collected for analysis. CZA susceptibility was tested by disk diffusion or broth micro 
dilution. Other antimicrobial susceptibility tests were performed with VITEK 2 compact system (Meniere, French), according to the manufacturer's instructions. Resistance to carbapenems in Enterobacterales was defined as imipenem and/or meropenem $\mathrm{MIC}>4 \mu \mathrm{g} / \mathrm{mL}$. The type of carbapenemase of all CRKP isolates was determined by PCR using the GeneXpert System. MICs were classified according to breakpoints established by the Clinical and Laboratory Standards Institute (CLSI M100). Methods used for microbial identification and antibiotic sensitivity analysis did not change during the study period.

\section{Statistical Analysis}

Continuous variables were expressed either as mean \pm standard deviation (SD) or median and interquartile range (IQR) according to whether the distribution of the variables was normal or non-normal, and compared using the Student's $t$-test or Mann-Whitney $U$-test. Variables with a $P$ value $<0.05$ (two-tailed) were considered statistically significant. All statistical analyses were performed by the SPSS20.0.

\section{Results}

\section{Clinical and Microbiological Characteristics}

There were overall 72 LT recipients diagnosed with CRE infections during the study period, 21 patients with CRKP infection were included in our study from April 2018 to August 2021. Most of the strains were KPC-Kp (17/21, $81 \%)$. The mean age was 40.47 years and $66.7 \%(14 / 21)$ were male. The mean MELD score was 20.8 in patients older than 12 years, and PELD score was 30.7 in patients younger than 12 years. Four $(4 / 21,19 \%)$ patients treated with CZA were pediatric liver transplant recipients. Most of the participants received CZA therapy for KPC-Kp infection. Clinical and demographic characteristics of the patients are summarized in Table 1. The etiology of primary liver disease included acute-on-chronic liver failure $(n=7,33.3 \%)$, HBV or HCV-related cirrhosis $(n=4,19 \%)$, hepatocellular carcinoma $(n=4,19 \%)$, polycystic liver disease $(n=1,4.8 \%)$ and Wilson's disease $(n=1,4.8 \%)$. The etiology of primary liver disease for the pediatric liver transplant patients was biliary atresia.

The most frequent source of infection was intraabdominal $(n=12)$ (including the four pediatric patients), followed by bloodstream infection $(n=8)$, pulmonary infection $(n=7)$, catheter-related bacteremia $(n=2)$, and seven patients presented with polymicrobial infection and two with septic shock. The mean SOFA score was 11.1 in patients due to the CRKP infection after liver transplantation. All patients with abdominal infection were treated with source control of abdominal drainage.

Based on CLSI criteria, all 21 pathogens displayed in vitro susceptibility to CZA, while they were all resistant to penicillins, extended-spectrum cephalosporins and ciprofloxacin, and minimum inhibitory concentrations (MICs) of imipenem were more than $16 \mu \mathrm{g} / \mathrm{mL}$. As such, some isolates were susceptible to polymyxin B (20/21, $95.2 \%)$ tigecycline $(19 / 21,90.5 \%)$, Trimethoprim-sulfamethoxazole $(16 / 21,76.2 \%)$ and amikacin (11/21, $52.4 \%$ ). Antimicrobial susceptibility of isolates is shown in Table 2.

\section{Antibiotic Regimens for CRKP}

Prior to CZA treatment, the median (IQR) time of treatment with other agents in 21 patients was 10 days (4-31.5 days). The most common regimes prescribed prior to initiation of CZA were carbapenems $(n=14)$, tigecycline $(n=9)$, vancomycin $(n=9)$ and cefoperazone-sulbactam $(n=9)$. CZA was administered as monotherapy in 7 patients $(7 / 21,33.3 \%)$ and combination regimens in $14(14 / 21$, $66.7 \%$ ) (including two pediatric patients), with meropenem $(n=5)$, aztreonam $(n=5)$, metronidazole $(n=4)$, aztreonam and metronidazole $(n=1)$, vancomycin and levofloxacin $(n=1)$, polymyxin $B$, vancomycin and levofloxacin $(n=1)$. In these 21 patients with CZA therapy, three cases developed CRKP recurrence 30 days after the cessation of CZA, and then retreated with CZA plus polymyxin B and levofloxacin or aztreonam. Patients with combination therapy showed more pulmonary infection than those with monotherapy $(35.7 \%, 5 / 14$ vs $28.6 \%, 2 /$ 7 ), and also higher incidence with severe sepsis $(21.4 \%, 3 /$ 14 vs $0 \%, 0 / 7)$. Median number of days from the onset of CRKP infection until the initiation of CZA salvage treatment was 2 days (IQR, 1-6.5), and median duration of CZA was 12 days (IQR, 8.5-19).

\section{Clinical Outcomes}

Among the 21 patients treated with CZA, the primary outcome of 30-day clinical response after CZA onset was reached $61.9 \%(13 / 21)$, respectively. Clinical response of 14-day and 30-day in adult patients was 70.6\% (12/17) and $58.8 \%(10 / 17)$, respectively, while in pediatric patients, the 14-day and 30-day clinical response were $75 \%$, respectively. 


\begin{tabular}{|c|c|c|c|c|c|c|c|c|c|c|c|c|}
\hline$\stackrel{\circ}{0}$ & $\stackrel{\varrho}{\Delta}$ & $\stackrel{\circ}{\Delta}$ & $\stackrel{\circ}{\Delta}$ & 总 & $\stackrel{\circ}{\Delta}$ & $\begin{array}{l}\stackrel{0}{0} \\
\stackrel{0}{\underline{\underline{i}}} \\
\underline{\underline{\varepsilon}}\end{array}$ & $\frac{0}{\stackrel{0}{0}}$ & $\frac{\stackrel{0}{0}}{\underline{\underline{\underline{a}}}}$ & $\check{\square}$ & $\stackrel{\circ}{\Delta}$ & 产 & $\stackrel{\frac{0}{0}}{\frac{0}{2}}$ \\
\hline $\bar{\Sigma} 0$ & a & $\therefore$ & ш & a & a & ш & ш & ш & ш & a & ш & ш \\
\hline 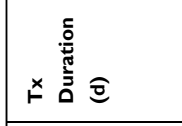 & 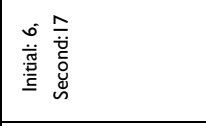 & in & $\underline{\underline{a}}$ & $\infty$ & 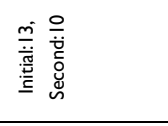 & 。 & $=$ & a & $\sigma$ & 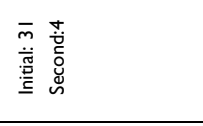 & $\underline{m}$ & 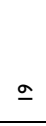 \\
\hline & 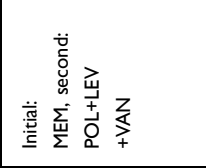 & $\underset{\text { 㭊 }}{\Sigma}$ & $\underset{\mathrm{w}}{\Sigma}$ & 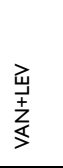 & 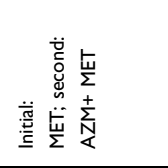 & 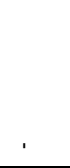 & 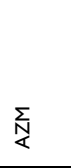 & $\underset{\mathbb{K}}{\Sigma}$ & . & 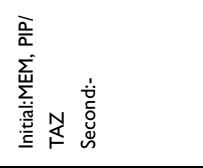 & $\underset{\mathbb{N}}{\Sigma}$ & $\underset{\mathrm{w}}{\Sigma}$ \\
\hline 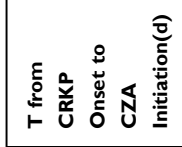 & 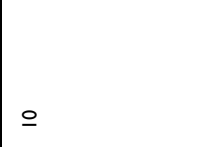 & o & $m$ & 으 & - & n & - & $\circ$ & - & - & - & $\sim$ \\
\hline 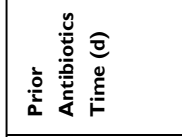 & $\tilde{m}$ & m & n & $\underline{m}$ & $\stackrel{m}{\circ}$ & $\underline{\underline{a}}$ & $\bar{m}$ & $\sigma$ & + & 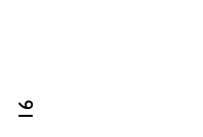 & 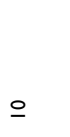 & m \\
\hline $\begin{array}{l}\frac{n}{n} \\
\frac{0}{0} \\
i n\end{array}$ & 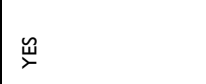 & 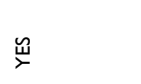 & $\stackrel{o}{z}$ & $\stackrel{0}{z}$ & $\stackrel{o}{z}$ & $\stackrel{o}{z}$ & $\stackrel{\circ}{z}$ & $\stackrel{o}{z}$ & o & 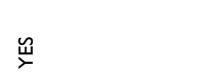 & $\stackrel{\bigcirc}{z}$ & $\stackrel{\circ}{z}$ \\
\hline 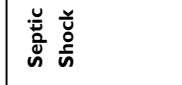 & 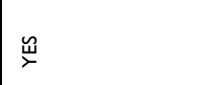 & $\stackrel{\circ}{z}$ & o & $\stackrel{\circ}{z}$ & $\stackrel{\circ}{z}$ & $\stackrel{\varrho}{z}$ & $\stackrel{\circ}{z}$ & $\stackrel{\circ}{z}$ & $\stackrel{\circ}{z}$ & $\stackrel{o}{z}$ & $\stackrel{0}{z}$ & $\stackrel{\circ}{z}$ \\
\hline 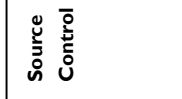 & 迎 & 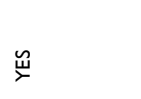 & $\stackrel{\circ}{z}$ & $\stackrel{\circ}{z}$ & 巡 & $\stackrel{q}{z}$ & 送 & $\underset{\nu}{\overleftrightarrow{\nu}}$ & o & 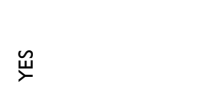 & $\stackrel{0}{z}$ & 送 \\
\hline 总 & 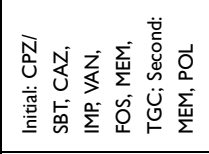 & 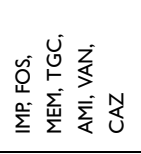 & N & 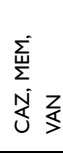 & 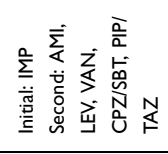 & 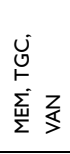 & 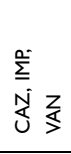 & 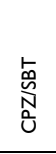 & 䟢 & 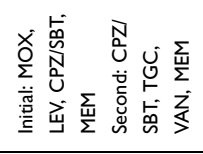 & 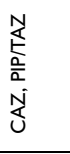 & $\begin{array}{l}\text { 岁 } \\
\text { N } \\
\text { Ũ }\end{array}$ \\
\hline 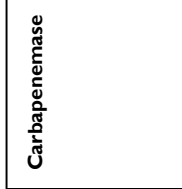 & $\begin{array}{l}\tilde{u} \\
\dot{y}\end{array}$ & $\begin{array}{l}\tilde{U} \\
\dot{z}\end{array}$ & $\begin{array}{l}\tilde{u} \\
\frac{u}{y}\end{array}$ & $\begin{array}{l}\tilde{U} \\
\underline{\Sigma}\end{array}$ & $\begin{array}{l}\tilde{u} \\
\underline{z}\end{array}$ & $\begin{array}{l}\tilde{u} \\
\underline{x}\end{array}$ & $\begin{array}{l}\tilde{u} \\
\underline{u}\end{array}$ & $\begin{array}{l}\tilde{u} \\
\frac{u}{y}\end{array}$ & $\begin{array}{l}\tilde{U} \\
\dot{v}\end{array}$ & $\begin{array}{l}\tilde{u} \\
\underline{y}\end{array}$ & $\begin{array}{l}\tilde{U} \\
\dot{u}\end{array}$ & $\begin{array}{l}\tilde{U} \\
\dot{\alpha} \\
\underline{y}\end{array}$ \\
\hline 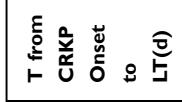 & - & ธ & $m$ & - & 另 & $\circ$ & $m$ & $\stackrel{\circ}{n}$ & $\bar{\infty}$ & $m$ & n & $m$ \\
\hline 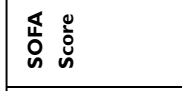 & $\underline{m}$ & $\simeq$ & $=$ & $\underline{\underline{n}}$ & $\infty$ & N & \pm & $\underline{\underline{ }}$ & in & $\underline{n}$ & \pm & $=$ \\
\hline 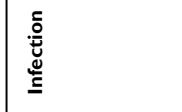 & $\begin{array}{l}\dot{\vec{\Delta}} \\
\overline{\overrightarrow{\mathrm{a}}} \overline{\mathrm{a}}\end{array}$ & $\begin{array}{l}\bar{\Xi} \\
\overline{\bar{a}}\end{array}$ & $\overline{\check{\alpha}}$ & $\bar{\alpha}$ & $\begin{array}{l}\bar{a} \\
\dot{\underline{s}}\end{array}$ & 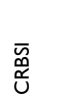 & 富 & $\bar{\Xi}$ & $\overline{\mathrm{s}}$ & $\begin{array}{l}\dot{\vec{a}} \\
\overrightarrow{\bar{\Delta}} \bar{a}\end{array}$ & $\overline{\mathrm{a}}$ & 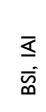 \\
\hline 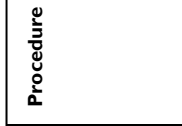 & 志 & 吉 & 吉 & 志 & 吉 & 吉 & t̆ & 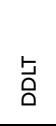 & 吉 & 吉 & 茄 & 芯 \\
\hline 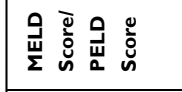 & $\underline{n}$ & n & $=$ & $\stackrel{m}{m}$ & $\stackrel{\infty}{\sim}$ & 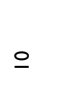 & $\bar{s}$ & $\underline{\infty}$ & $=$ & 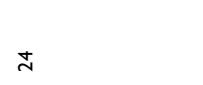 & $\widetilde{m}$ & $\bar{\sigma}$ \\
\hline 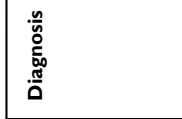 & 竞 & $\stackrel{U}{\text { U }}$ & 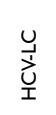 & 岂 & 岂 & $\frac{9}{a}$ & 嵌是 & 岁 & 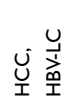 & 岂 & 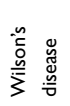 & 晏 \\
\hline 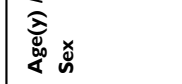 & $\underset{\text { क्ष }}{\sum}$ & $\sum_{y}$ & $\sum_{\substack{\mathrm{j} \\
\mathrm{j}}}$ & $\underset{m}{\sum}$ & $\sum_{j}$ & $\frac{\vec{u}}{\sigma}$ & $\sum_{0}^{\Sigma}$ & 立 & $\sum_{\text {క }}$ & $\underset{\mathbb{S}}{\Sigma}$ & 屵 & $\sum_{\forall}$ \\
\hline$\dot{\mathbf{z}}$ & - & N & $m$ & + & in & $\circ$ & n & $\infty$ & $\sigma$ & $\circ$ & $=$ & $\simeq$ \\
\hline
\end{tabular}




\begin{tabular}{|c|c|c|c|c|c|c|c|c|c|}
\hline 帝 & $\stackrel{\circ}{\circ}$ & 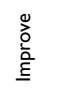 & $\stackrel{\circ}{\circ}$ & 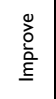 & $\stackrel{\circ}{\Delta}$ & $\begin{array}{l}\frac{0}{0} \\
\frac{0}{\underline{0}} \\
\underline{\underline{\underline{E}}}\end{array}$ & 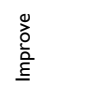 & 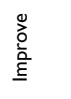 & 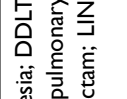 \\
\hline ш & a & ш & a & ш & a & ш & ш & ш & 势 \\
\hline$=$ & $\infty$ & $\underline{m}$ & 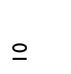 & $\sigma$ & m & $\underline{\infty}$ & \pm & n & 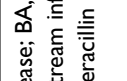 \\
\hline . & & 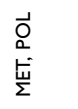 & $\stackrel{\breve{\Sigma}}{\Sigma}$ & . & . & 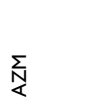 & 岀 & . & 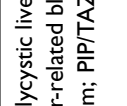 \\
\hline+ & N & - & m & - & $\stackrel{\text { i }}{ }$ & $\tilde{m}$ & m & $\sigma$ & 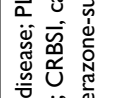 \\
\hline in & $\underline{m}$ & in & $\bar{m}$ & in & $\hat{\lambda}$ & m & m & m & 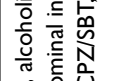 \\
\hline о & $\stackrel{\circ}{z}$ & $\stackrel{\bigcirc}{z}$ & $\stackrel{\bigcirc}{z}$ & $\stackrel{\circ}{z}$ & $\stackrel{\bigcirc}{z}$ & $\stackrel{\circ}{z}$ & $\stackrel{\circ}{z}$ & $\stackrel{\circ}{z}$ & \\
\hline ’ & $\stackrel{\circ}{z}$ & $\stackrel{\bigcirc}{z}$ & $\stackrel{\circ}{z}$ & $\stackrel{\circ}{z}$ & $\ddot{\ddot{\nu}}$ & $\stackrel{O}{z}$ & $\stackrel{\rho}{z}$ & $\stackrel{\circ}{z}$ & \\
\hline ’o & $\stackrel{\circ}{z}$ & $\stackrel{\circ}{z}$ & 亗 & $\stackrel{\circ}{z}$ & $\ddot{\ddot{\nu}}$ & $\ddot{\ddot{\nu}}$ & $\ddot{\ddot{x}}$ & $\stackrel{\mathscr{P}}{\check{2}}$ & \\
\hline 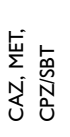 & 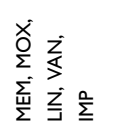 & 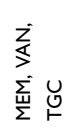 & 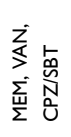 & 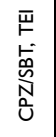 & 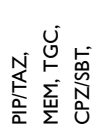 & 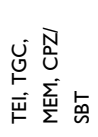 & 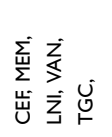 & 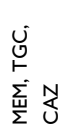 & 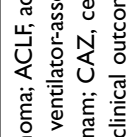 \\
\hline $\begin{array}{l}\tilde{U} \\
\dot{u}\end{array}$ & $\begin{array}{l}\tilde{U} \\
\dot{v}\end{array}$ & $\begin{array}{l}\tilde{u} \\
\dot{y}\end{array}$ & $\frac{\bar{\lambda}}{\grave{z}}$ & $\begin{array}{l}\tilde{u} \\
\underline{y}\end{array}$ & $\frac{\bar{\lambda}}{\grave{z}}$ & $\frac{\bar{T}}{\dot{\Sigma}}$ & $\begin{array}{l}\tilde{U} \\
\dot{y}\end{array}$ & $\frac{\bar{T}}{\dot{\Sigma}}$ & 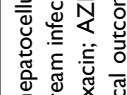 \\
\hline+ & f & $m$ & 崩 & n & $m$ & n & बे & n & \\
\hline$\infty$ & $\sigma$ & $\alpha$ & $\underline{m}$ & $=$ & $\simeq$ & $\sigma$ & + & - & \\
\hline ডু & $\bar{\alpha}$ & $\overline{\mathrm{a}}$ & $\underline{\Xi}$ & $\overline{\check{~}}$ & $\underset{\bar{a}}{\bar{a}}$ & $\Xi$ & $\bar{\Xi}$ & $\bar{\Phi}$ & 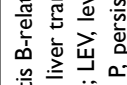 \\
\hline 志 & '⿳亠口冋口 & t⿳亠口冋口 & 吉 & 吉 & 吉 & 吉 & 志 & 吉 & \\
\hline$\infty$ & $\circ$ & \pm & i & $\stackrel{m}{m}$ & $\approx$ & n & $\stackrel{\infty}{\sim}$ & ธ & 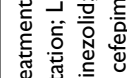 \\
\hline$\stackrel{\breve{Y}}{\underline{I}}$ & $\stackrel{\breve{Y}}{\underline{x}}$ & 岂 & 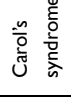 & 岂 & $\Phi$ & ه్ & ऽ & ऽ & $\mathbb{E}$ \\
\hline 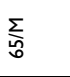 & $\sum_{\text {S }}$ & 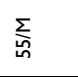 & $\underset{\underline{\sigma}}{\underline{\Sigma}}$ & $\sum_{\bar{j}}$ & $\stackrel{\Perp}{ \pm}$ & $\stackrel{u}{N}$ & $\stackrel{\underline{u}}{\underline{u}}$ & 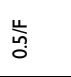 & \\
\hline$\underline{m}$ & \pm & $\underline{n}$ & $\underline{0}$ & $\simeq$ & $\underline{\infty}$ & $\underline{a}$ & i & $\bar{\sim}$ & \\
\hline
\end{tabular}


Table 2 Susceptibility Testing Results of Klebsiella pneumoniae Isolates to Antimicrobial Agents

\begin{tabular}{|l|l|l|l|}
\hline \multirow{2}{*}{ Antimicrobial Agent } & \multicolumn{3}{|c|}{ Klebsiella pneumoniae (n=2I) } \\
\cline { 2 - 4 } & S & I & R \\
\hline Amikacin & $11(52.4 \%)$ & 0 & $10(47.6 \%)$ \\
\hline Ampicillin & 0 & 0 & $21(100 \%)$ \\
\hline Piperacillin-tazobactam & 0 & 0 & $21(100 \%)$ \\
\hline Imipenem & 0 & 0 & $21(100 \%)$ \\
\hline Meropenem & 0 & 0 & $21(100 \%)$ \\
\hline Cefoperazone-sulbactam & 0 & $1(4.8 \%)$ & $20(95.2 \%)$ \\
\hline Cefepime & 0 & 0 & $21(100 \%)$ \\
\hline Aztreonam & 0 & 0 & $21(100 \%)$ \\
\hline Levofloxacin & 0 & 0 & $21(100 \%)$ \\
\hline Trimethoprim-sulfamethoxazole & $16(76.2 \%)$ & 0 & $9(42.8 \%)$ \\
\hline Tigecycline & $19(90.5 \%)$ & 0 & $2(9.5 \%)$ \\
\hline Polymyxin B & $20(95.2 \%)$ & 0 & $1(4.8 \%)$ \\
\hline Ceftazidime Avibactam & $21(100 \%)$ & 0 & 0 \\
\hline
\end{tabular}

Six cases of 14-day clinical failure were attributed to organ failure $(n=4)$ and uncontrolled infection $(n=2)$. Of the eight patients with 30-day clinical failure was due to CRKP recurrence in the same location $(n=2)$, persistence of symptoms and signs of infection $(n=3)$ and organ failure $(\mathrm{n}=3)$, but 2 patients showed a 14-day clinical response. The all-cause mortality rate was $42.9 \%(\mathrm{n}=9 / 21)$, including one child. Infection-related all-cause mortality was $83.3 \%(5 / 9)$, comprising three cases with recurrent intraabdominal and pulmonary infection and two cases with uncontrolled pulmonary infection, while gastrointestinal hemorrhage contributed $11.1 \%$ of all-cause mortality in one patient, three others died from organ failure. The nine patients who experienced clinical failure are summarized in Table 3.

\section{Microbiology Outcomes}

Microbiologic failures occurred in $42.9 \%(9 / 21)$ of episodes due to relapsing CRKP infections $(n=3)$, death $(n=4)$ and respiratory colonization $(\mathrm{n}=2)$. The recurrence episode happened in the same location as the previous. One case developed a second intra-abdominal infection 34 days after CZA monotherapy, and retreated with CZA combined with aztreonam. One case presented a relapse in pulmonary after 30 days of completing infection with
CZA monotherapy, and retreated with CZA combined with polymyxin B. Another case was relapsed in pulmonary after 44 days of combination with meropenem, and retreated with CZA monotherapy. In these cases, the CRKP isolates remained susceptible to CZA, although the clinical and microbiological cures were failure after retreatment with CZA monotherapy or plus with other agents.

\section{Adverse Events}

Three patients develop acute renal failure, perhaps due to progressive infection during treatment period $(285 \mathrm{umol} / \mathrm{L}$ to $451 \mathrm{umol} / \mathrm{L}, 81 \mathrm{umol} / \mathrm{L}$ to $823 \mathrm{umol} / \mathrm{L}$ and $36 \mathrm{umol} / \mathrm{L}$ to $243 \mathrm{umol} / \mathrm{L}$, respectively), and two patients received hemodialysis after CZA treatment. One of these patients had combined polymyxin B treatment, which primarily contributed to renal dysfunction.

Two patients developed alanine aminotransferase (ALT) $(67 \mathrm{U} / \mathrm{L}$ to $189 \mathrm{U} / \mathrm{L}$ and $17 \mathrm{U} / \mathrm{L}$ to $152 \mathrm{U} / \mathrm{L})$ and aspartate aminotransferase (AST) $(34 \mathrm{U} / \mathrm{L}$ to $99 \mathrm{U} / \mathrm{L}$ and $25 \mathrm{U} / \mathrm{L}$ to $77 \mathrm{U} / \mathrm{L}$ ) increased, one concomitant with tigecycline, and the other with voriconazole. No adverse reactions occurred in the skin, subcutaneous tissue, gastrointestinal system or nervous system in our study.

For the four pediatric recipients, no adverse events such as renal dysfunction, rash, diarrhea, vomiting or neurotoxicity were found in this study.

\section{Discussion}

This case series study is firstly evaluating effectiveness of treatment with CZA in LT recipients due to infection with CRKP. Even with the limitation of small sample size, it demonstrated the clinical benefit of CZA for the treatment of CRE infection. In our experience, the 14-day and 30day mortality rates were $28.6 \%$ and $38.1 \%$, respectively, consistent with a recently published report which observed a $34.1 \%$ of 30-day mortality. ${ }^{22}$ In Tumbarello et al's study, the 30 -day all-cause mortality was $25.3 \%$, the mortality between patients managed with CZA alone and those treated with combination was no statistically significant difference, but CZA administration by prolonged infusion significantly reduced mortality. ${ }^{29}$ It is also in line with the all-cause in-hospital mortality rate of $39.5 \%$ reported in other patients who were managed with CZA salvage therapy. ${ }^{30}$ However, in a recent prospective cohort study, the group of patients (96\% of them with KPC-Kp infections) received first-line treatment with CZA, a very low 30-day mortality rate was observed $(8 \%) .{ }^{31}$ Compared 
Table 3 Description of Patients Infected by Carbapenem-Resistant Klebsiella Pneumoniae Who Experienced Clinical Failure

\begin{tabular}{|c|c|c|c|c|c|c|c|c|}
\hline $\begin{array}{l}\text { Agel } \\
\text { Sex }\end{array}$ & $\begin{array}{l}\text { Underlying } \\
\text { Condition }\end{array}$ & $\begin{array}{l}\text { Type of } \\
\text { Infection }\end{array}$ & $\begin{array}{l}\text { Clinical } \\
\text { Presentation }\end{array}$ & Prior Therapy to CZA & $\begin{array}{l}\text { Dose of } \\
\text { CZA }\end{array}$ & CRRT & $\begin{array}{l}\text { Combined } \\
\text { Antibiotics }\end{array}$ & $\begin{array}{l}\text { Reason for } \\
\text { Clinical } \\
\text { Failure }\end{array}$ \\
\hline $48 / M$ & HBV-LC & $\begin{array}{l}\text { BSI, IAI, } \\
\text { HAP }\end{array}$ & $\begin{array}{l}\text { Septic shock, } \\
\text { sepsis }\end{array}$ & $\begin{array}{l}\text { Initial: CPZ/SBT for 9d, } \\
\text { IMP/VAN for } 5 d, \text { TGC / } \\
\text { MEM/FOS for } 6 d \text {, Second: } \\
\text { MEM/POL for } 30 d\end{array}$ & $\begin{array}{l}\text { Intial:2.5g } \\
\text { qI2h for } 6 \mathrm{~d} \text {; } \\
\text { Second: } 1.25 \mathrm{~g} \\
\text { qI2h for } 17 \mathrm{~d}\end{array}$ & YES & $\begin{array}{l}\text { Initial: MEM; } \\
\text { Second: } \\
\text { POL+ LEV }\end{array}$ & $\begin{array}{l}\text { Recurrent } \\
\text { infection; } \\
\text { Death }\end{array}$ \\
\hline $45 / M$ & $\mathrm{HCC}$ & BSI, IAI & Sepsis & $\begin{array}{l}\text { IMP for } 4 d, \text { MEM/FOS / } \\
\text { TGC for } 18 \mathrm{~d}, \text { IMP/FOS / } \\
\text { TGC for } 10 \mathrm{~d}\end{array}$ & $\begin{array}{l}2.5 \mathrm{~g} q 12 \mathrm{~h} \text { for } \\
5 \mathrm{~d}\end{array}$ & NO & MEM & $\begin{array}{l}\text { Respiratory } \\
\text { failure, Death }\end{array}$ \\
\hline $54 / M$ & $\begin{array}{l}\text { HCV-LC } \\
\text { UGIB }\end{array}$ & BSI & No sepsis & CAZ for Id & $\begin{array}{l}2.5 \mathrm{~g} \mathrm{q} / 2 \mathrm{~h} \text { for } \\
19 \mathrm{~d}\end{array}$ & YES & MEM & $\begin{array}{l}\text { Gastrointestinal } \\
\text { bleeding, } \\
\text { Hemorrhagic } \\
\text { shock, Death }\end{array}$ \\
\hline $4 I / M$ & HBV-LC & $\begin{array}{l}\text { IAI } \\
\text { HAP }\end{array}$ & No sepsis & $\begin{array}{l}\text { Initial: IMP for } 2 d \text {, Second: } \\
\text { AMI/CPZ/SBT for I7d, } \\
\text { LEV 7d, VAN /PIP/TAZ for } \\
\text { I0d }\end{array}$ & $\begin{array}{l}\text { Initial: } 2.5 \mathrm{~g} \\
\mathrm{q} / 2 \mathrm{~h} \text { for } 4 \mathrm{~d} \text {; } \\
\text { Second:2.5g } \\
\mathrm{q} / 2 \mathrm{~h} \text { for } 7 \mathrm{~d}\end{array}$ & NO & $\begin{array}{l}\text { Initial:MET; } \\
\text { Second: } \\
\text { AZM+MET }\end{array}$ & $\begin{array}{l}\text { Recurrent } \\
\text { infection; } \\
\text { Death }\end{array}$ \\
\hline $65 / M$ & $\begin{array}{l}\text { HBV-LC, } \\
\text { HCC }\end{array}$ & BSI & No sepsis & CAZ for Id & $\begin{array}{l}2.5 \mathrm{~g} q 12 \mathrm{~h} \text { for } \\
10 \mathrm{~d}\end{array}$ & NO & NO & $\begin{array}{l}\text { Hepatic failure, } \\
\text { Death }\end{array}$ \\
\hline 68/F & ACLF & $\begin{array}{l}\text { BSI } \\
\text { IAI } \\
\text { PI }\end{array}$ & Sepsis & $\begin{array}{l}\text { Initial: MOX for } 5 d \text {, LEV } \\
\text { for } 2 d, \text { CPZ/SBT for } 4 d \text {, } \\
\text { MEM for } 20 \mathrm{~d} \\
\text { Second: PIP/TAZ for 6d, } \\
\text { CPZ/SBT for 14d, TGC } \\
\text { for 19d, VAN for 16d, } \\
\text { MEM for } 16 \mathrm{~d}\end{array}$ & $\begin{array}{l}\text { Initial: } 2.5 \mathrm{~g} q 8 \mathrm{~h} \\
\text { for } 6 \mathrm{~d}, 2.5 \mathrm{~g} \\
\mathrm{q} 12 \mathrm{~h} \text { for } 25 \mathrm{~d} \text {; } \\
\text { Second: } 2.5 \mathrm{~g} \\
\mathrm{q} 12 \mathrm{~h} \text { for } 4 \mathrm{~d}\end{array}$ & NO & $\begin{array}{l}\text { Initial:MEM, } \\
\text { PIP/TAZ; } \\
\text { Second: NO }\end{array}$ & $\begin{array}{l}\text { Multiple organ } \\
\text { failure, } \\
\text { Recurrent } \\
\text { infection, Death }\end{array}$ \\
\hline $59 / M$ & $\mathrm{HCC}$ & HAP & No sepsis & $\begin{array}{l}\text { MEM for } 3 d, \text { MOX for } 9 d \text {, } \\
\text { LIN for } 3 d \text {, VAN for } 8 d \text {, } \\
\text { IMP for } 2 d\end{array}$ & $\begin{array}{l}2.5 \mathrm{gq} q \mathrm{~h} \text { for } \\
8 \mathrm{~d}\end{array}$ & NO & NO & $\begin{array}{l}\text { Severe } \\
\text { pulmonary } \\
\text { infection, } \\
\text { Gastrointestinal } \\
\text { bleeding, Death }\end{array}$ \\
\hline I8/M & LC & $|A|$ & No sepsis & $\begin{array}{l}\text { MEM for 20d, VAN for } \\
28 d, \text { CPZ/SBT for } 8 d\end{array}$ & $\begin{array}{l}2.5 \mathrm{~g} \mathrm{q} 8 \mathrm{~h} \text { for } \\
10 \mathrm{~d}\end{array}$ & NO & MET & $\begin{array}{l}\text { Recurrent } \\
\text { infection, Severe } \\
\text { pulmonary } \\
\text { infection, Death }\end{array}$ \\
\hline $\mathrm{I} / \mathrm{F}$ & $\mathrm{BA}$ & $\begin{array}{l}\text { HAP } \\
\text { IAI }\end{array}$ & Septic shock & $\begin{array}{l}\text { PIP/TAZ for 3d, MEM for } \\
22 d, \text { TGC for } 24 d, C P Z I \\
\text { SBT for } 5 d\end{array}$ & $\begin{array}{l}0.3 \mathrm{gq} q \mathrm{~h} \text { for } \\
3 \mathrm{~d}\end{array}$ & NO & NO & $\begin{array}{l}\text { Multiple organ } \\
\text { failure, Septic } \\
\text { shock, Death }\end{array}$ \\
\hline
\end{tabular}

Abbreviations: IMP, imipenem; MEM, meropenem; TEI, teicoplanin; TMP-SMZ, trimethoprim-sulfamethoxazole; VAN, vancomycin; TGC, tigecycline; AMI, amikacin; LIN, linezolid; POL, polymyxin; LEV, levofloxacin; AZM, aztreonam; CAZ, ceftazidime; MET, metronidazole; CPZ/SBT, cefoperazone-sulbactam; PIP/TAZ, piperacillin and tazobactam.

with our series, CZA was started in most of LT patients owing to failure of previous anti-bacterial regimens.

The rapid onset of CZA resistance was the most concerning events by Shields et al, which reporting a rate of recurrences in $17 \%(5 / 37)$ patients and a rate of $10 \%(8 /$
77) patients developed CZA resistance after a median of 15 days of CZA treatment. ${ }^{26}$ Another study also showed $13.5 \%(5 / 37)$ patients had experienced recurrence. ${ }^{21}$ Consistent to these studies, in our series we found 3 patients with intra-abdominal and pulmonary infection 
observed recurrence in the same location. We did not observe CRKP isolates acquiring in vitro resistance to CZA during or after treatment. Indeed, no resistant CRE strains have been isolated in our center so far, although other studies have demonstrated that the KPC-3 or KPC-2 strains have developed resistance to $\mathrm{CZA} .^{21,26,30}$

In accordance with previous reports, CZA combination therapy was not associated with a reduced risk of clinical failure. $^{21,22,25,26}$ Among 14 CZA-based combination group in our cohort, eight patients died. However, we cannot draw a definitive conclusion as for the small samples. One of explanations maybe that the patients who received CZA combination therapy suffered more severe infection than those patients who did not. These data are in contrast to previous studies which showed that the combination of two or more in vitro active drugs was associated with lower mortality than monotherapy, especially in the most of patients with the most severe infections. ${ }^{14,32,33} \mathrm{CZA}$ monotherapy presumably associated with decreasing the rate of adverse events, particularly acute kidney injury, which frequently emerged during the treatments based on combinations with aminoglycosides or colistin. ${ }^{21,33}$ In our series, three patients developed acute renal failure, and two patients received hemodialysis during the treatment. All these three patients received combination therapy with meropenem $(n=2)$ or polymyxin $B(n=1)$. In contrast, renal impairment was not detected during or after the treatment with CZA. Further studies should be conducted to determine whether combination therapy with CZA could improve prognosis of patients with serious CRKP infections.

In our study, four pediatric liver transplantation patients were treated with CZA for abdominal infection due to CRKP, with a mortality of $25 \%$. CZA was expanded the approval to include pediatric patients aged $\geq 3$ months by the FDA for treatment of complicated intra-abdominal infection as well as complicated urinary tract infections, including pyelonephritis, used in combination with metronidazole till March 2019. However, safety and effectiveness have not been evaluated in pediatric liver transplantation patients with CRKP infectious. The recent INFORM Surveillance program (USA 2011-2015) reported a highly in vitro-activity of ceftazidime/avibactam against $K$. pneumoniae isolated from pediatric patients. $^{34}$ In this study, a total of 8461 gram-negative strains collected from hospitalized pediatric patients in the United States Medical Center were confirmed to ceftazidime avibactam was in vitro activity against $>99.9 \%$ of
Enterobacterales and $99.1 \%$ of $P$. aeruginosa strains. In a randomized Phase II study of 95 patients aged 3 months to 18 years with complicated urinary tract infection (cUTI), CZA was well tolerated in these children and the safety profile was in line with ceftazidime monotherapy in pediatric patients. Also, CZA appeared effective in the treatment of pediatric cUTI caused by Gram-negative pathogens, with favorable clinical and microbiologic response rates observed against the predominant cUTI pathogen (E. coli), including ceftazidime-non-susceptible isolates. ${ }^{35}$ Although CZA has shown safety and efficacy in pediatric liver transplantation patients in our study, however, it is impossible to draw a specific conclusion due to the small number of pediatric liver transplantation patients in this study, more clinical studies were needed to confirm the results.

This study had several limitations by the small samples and retrospective design, which hindered the ability to make definitive conclusion about utility of LT patients with CRKP infection by CZA. On the other hand, all of patients received CZA as salvage therapy; hence, it may not be generalized to patients who can get first-line treatment of CZA. Nonetheless, our experience suggested CZA may be an available alternatively in the treatment of CRKP infections in advance and with less toxic.

In conclusion, data on this study indicate that CZA is a promising option, even in monotherapy, for the treatment of patients with severe infections due to Carbapenemresistant $K$. pneumoniae among LT recipients. The emergence of resistance to CZA was not observed.

\section{Funding}

1. WU JIEPING Medical Foundation (320.6750.2020-04$31)$; 2. China International Medical Foundation (Z-201835-2003).

\section{Disclosure}

The authors report no conflicts of interest in this work.

\section{References}

1. Lübbert C, Becker-Rux D, Rodloff AC, et al. Colonization of liver transplant recipients with KPC-producing Klebsiella pneumoniae is associated with high infection rates and excess mortality: a case-control analysis. Infection. 2014;42(2):309-316. doi:10.1007/ s15010-013-0547-3

2. Giannella M, Bartoletti M, Morelli MC, et al. Risk factors for infection with carbapenem-resistant Klebsiella pneumoniae after liver transplantation: the importance of pre- and posttransplant colonization. Am J Transplant. 2015;15(6):1708-1715. doi:10.1111/ajt.13136 
3. Carrion AF. Risk factors and outcomes of carbapenem-resistant Klebsiella pneumoniae infections in liver transplant recipients. Liver Transplant. 2016;22(5):698-699. doi:10.1002/lt.24413

4. Huprikar S. Update in infectious diseases in liver transplant recipients. Clin Liver Dis. 2007;11(2):337-354. doi:10.1016/j.cld.2007.04.006

5. Varghese J, Jayanthi V, Rela M. Mortality associated with carbapenem-resistant Klebsiella pneumoniae infections in liver transplant recipients. Liver Transplant. 2012;18(9):1124;author reply 1125. doi:10.1002/lt.23464

6. Linares L, Cervera C, Hoyo I, et al. Klebsiella pneumoniae infection in solid organ transplant recipients: epidemiology and antibiotic resistance. Transplant Proc. 2010;42(8):2941-2943. doi:10.1016/j. transproceed.2010.07.080

7. Bergamasco MD, Barroso Barbosa M, de Oliveira Garcia D, et al. Infection with Klebsiella pneumoniae carbapenemase (KPC)-producing K. pneumoniae in solid organ transplantation. Transplant Infect Dis. 2012;14(2):198-205. doi:10.1111/j.1399-3062.2011.00688.x

8. Mouloudi E, Massa E, Piperidou M, et al. Tigecycline for treatment of carbapenem-resistant Klebsiella pneumoniae infections after liver transplantation in the intensive care unit: a 3-year study. Transplant Proc. 2014;46(9):3219-3221. doi:10.1016/j.transproceed.2014.09.160

9. Hand J, Patel G. Multidrug-resistant organisms in liver transplant: mitigating risk and managing infections. Liver Transplant. 2016;22 (8):1143-1153. doi:10.1002/lt.24486

10. Barchiesi F, Montalti R, Castelli P, et al. Carbapenem-resistant Klebsiella pneumoniae influences the outcome of early infections in liver transplant recipients. BMC Infect Dis. 2016;16(1):538 doi:10.1186/s12879-016-1876-5

11. Clancy CJ, Chen L, Shields RK, et al. Epidemiology and molecular characterization of bacteremia due to carbapenem-resistant Klebsiella pneumoniae in transplant recipients. Am J Transplant. 2013;13 (10):2619-2633. doi:10.1111/ajt.12424

12. Villegas MV, Pallares CJ, Escandón-Vargas K. Characterization and clinical impact of bloodstream infection caused by carbapenemase-producing Enterobacteriaceae in seven Latin American Countries. PLoS One. 2016;11(4):e0154092. doi:10.1371/ journal.pone.0154092

13. Kohler PP, Volling C, Green K, Uleryk EM, Shah PS, McGeer A. Carbapenem resistance, initial antibiotic therapy, and mortality in Klebsiella pneumoniae bacteremia: a systematic review and meta-analysis. Infect Control Hosp Epidemiol. 2017;38 (11):1319-1328. doi:10.1017/ice.2017.197

14. Falagas ME, Tansarli GS, Karageorgopoulos DE, Vardakas KZ. Deaths attributable to carbapenem-resistant Enterobacteriaceae infections. Emerg Infect Dis. 2014;20(7):1170-1175. doi:10.3201/eid2007.121004

15. Benattar YD, Omar M, Zusman O, et al. The effectiveness and safety of high-dose colistin: prospective cohort study. Clin Infect Dis. 2016;63(12):1605-1612. doi:10.1093/cid/ciw684

16. Liu YY, Wang Y, Walsh TR, et al. Emergence of plasmid-mediated colistin resistance mechanism MCR-1 in animals and human beings in China: a microbiological and molecular biological study. Lancet Infect Dis. 2016;16(2):161-168. doi:10.1016/S1473-3099(15)00424-7

17. Sheu CC, Chang YT, Lin SY, Chen YH, Hsueh PR. Infections caused by carbapenem-resistant Enterobacteriaceae: an update on therapeutic options. Front Microbiol. 2019;10:80. doi:10.3389/fmicb.2019.00080

18. Zasowski EJ, Rybak JM, Rybak MJ. The $\beta$-lactams strike back: ceftazidime-avibactam. Pharmacotherapy. 2015;35(8):755-770. doi:10.1002/phar.1622

19. Shirley M. Ceftazidime-avibactam: a review in the treatment of serious gram-negative bacterial infections. Drugs. 2018;78 (6):675-692. doi:10.1007/s40265-018-0902-x

20. Krapp F, Grant JL, Sutton SH, Ozer EA, Barr VO. Treating complicated carbapenem-resistant Enterobacteriaceae infections with ceftazidime/avibactam: a retrospective study with molecular strain characterisation. Int $J$ Antimicrob Agents. 2017;49(6):770-773. doi:10.1016/j.ijantimicag.2017.01.018
21. Shields RK, Potoski BA, Haidar G, et al. Clinical outcomes, drug toxicity, and emergence of ceftazidime-avibactam resistance among patients treated for carbapenem-resistant Enterobacteriaceae infections. Clin Infect Dis. 2016;63(12):1615-1618. doi:10.1093/cid/ciw636

22. Tumbarello M, Trecarichi EM, Corona A, et al. Efficacy of ceftazidime-avibactam salvage therapy in patients with infections caused by Klebsiella pneumoniae carbapenemase-producing $\mathrm{K}$. pneumoniae. Clin Infect Dis. 2019;68(3):355-364. doi:10.1093/cid/ciy492

23. Castón JJ, Lacort-Peralta I, Martín-Dávila P, et al. Clinical efficacy of ceftazidime/avibactam versus other active agents for the treatment of bacteremia due to carbapenemase-producing Enterobacteriaceae in hematologic patients. Int $J$ Infect Dis. 2017;59:118-123. doi:10.1016/j.ijid.2017.03.021

24. De la Calle C, Rodríguez O, Morata L, et al. Clinical characteristics and prognosis of infections caused by OXA-48 carbapenemase-producing Enterobacteriaceae in patients treated with ceftazidime-avibactam. Int J Antimicrob Agents. 2019;53 (4):520-524. doi:10.1016/j.ijantimicag.2018.11.015

25. Sousa A, Pérez-Rodríguez MT, Soto A, et al. Effectiveness of ceftazidime/avibactam as salvage therapy for treatment of infections due to OXA-48 carbapenemase-producing Enterobacteriaceae. J Antimicrob Chemother. 2018;73(11):3170-3175. doi:10.1093/jac/dky295

26. Shields RK, Nguyen MH, Chen L, Press EG, Kreiswirth BN, Clancy CJ. Pneumonia and renal replacement therapy are risk factors for ceftazidime-avibactam treatment failures and resistance among patients with carbapenem-resistant Enterobacteriaceae infections. Antimicrob Agents Chemother. 2018;62(5). doi:10.1128/AAC.02497-17

27. Horan TC, Andrus M, Dudeck MA. CDC/NHSN surveillance definition of health care-associated infection and criteria for specific types of infections in the acute care setting. Am J Infect Control. 2008;36 (5):309-332. doi:10.1016/j.ajic.2008.03.002

28. Castón JJ, Gallo M, García M, et al. Ceftazidime-avibactam in the treatment of infections caused by KPC-producing Klebsiella pneumoniae: factors associated with clinical efficacy in a single-center cohort. Int J Antimicrob Agents. 2020;56(3):106075. doi:10.1016/j. ijantimicag.2020.106075

29. Tumbarello M, Raffaelli F, Giannella M, et al. Ceftazidime-avibactam use for Klebsiella pneumoniae carbapenemase-producing K. pneumoniae infections: a retrospective observational multicenter study. Clin Infect Dis. 2021;73(9):1664-1676. doi:10.1093/cid/ciab176

30. Gutiérrez-Gutiérrez B, Salamanca E, de Cueto M, et al. Effect of appropriate combination therapy on mortality of patients with bloodstream infections due to carbapenemase-producing Enterobacteriaceae (INCREMENT): a retrospective cohort study. Lancet Infect Dis. 2017;17(7):726-734. doi:10.1016/S1473-3099(17)30228-1

31. Temkin E, Torre-Cisneros J, Beovic B, et al. Ceftazidime-avibactam as salvage therapy for infections caused by carbapenem-resistant organisms. Antimicrob Agents Chemother. 2017;61(2). doi:10.1128/AAC.01964-16

32. Tumbarello M, Trecarichi EM, De Rosa FG, et al. Infections caused by KPC-producing Klebsiella pneumoniae: differences in therapy and mortality in a multicentre study. J Antimicrob Chemother. 2015;70 (7):2133-2143. doi:10.1093/jac/dkv086

33. King M, Heil E, Kuriakose S, et al. Multicenter study of outcomes with ceftazidime-avibactam in patients with carbapenem-resistant Enterobacteriaceae infections. Antimicrob Agents Chemother. 2017;61(7). doi:10.1128/AAC.00449-17

34. Sader HS, Huband MD, Duncan LR, Flamm RK. Ceftazidime-avibactam antimicrobial activity and spectrum when tested against gram-negative organisms from pediatric patients: results from the INFORM surveillance program (United States, 2011-2015). Pediatr Infect Dis J. 2018;37 (6):549-554. doi:10.1097/INF.0000000000001859

35. Bradley JS, Roilides E, Broadhurst H, et al. Safety and efficacy of ceftazidime-avibactam in the treatment of children $\geq 3$ months to $<18$ years with complicated urinary tract infection: results from a phase 2 randomized, controlled trial. Pediatr Infect Dis J. 2019;38 (9):920-928. doi:10.1097/INF.0000000000002395 


\section{Publish your work in this journal}

Infection and Drug Resistance is an international, peer-reviewed openaccess journal that focuses on the optimal treatment of infection (bacterial, fungal and viral) and the development and institution of preventive strategies to minimize the development and spread of resistance. The journal is specifically concerned with the epidemiology of antibiotic resistance and the mechanisms of resistance development and diffusion in both hospitals and the community. The manuscript management system is completely online and includes a very quick and fair peerreview system, which is all easy to use. Visit http://www.dovepress.com/ testimonials.php to read real quotes from published authors. 